\title{
Referees for 2021
}

Published online: 18 November 2021

(c) IPNA 2021

Carolyn L. Abitbol

Pauline Abou Jaoudé

Fahd A. Ahmad

Ayse Akcan Arikan

Shreeram Akilesh

Abdullah Al Abbas

Demet Alaygut

Laura Fernanda Alconcher

R. Todd Alexander

Khalid A. Alhasan

Karel Allegaert

Sulaiman Al-Mayouf

Uri Alon

Amira Al-Uzri

Sandra Amaral

Elizabeth Ames

Sharon Andreoli

Ifeoma Anochie

M.S. Ansari

Victor Aquino

Gianluigi Ardissino

Gema Ariceta

Isa F. Ashoor

Meredith A. Atkinson

Bahriye Atmis

Christoph Aufricht

Midori Awazu

Rose Ayoob

Justine Bacchetta

Arvind Bagga

Sevcan A. Bakkaloglu

Alejandro Balestracci

Sushmita Banerjee

Rossana Baracco

Matthew F. Barhight

Biswanath Basu

Rajit Basu

Carlton M. Bates

Abbie Ruth Bauer
Michel G. Baum

Laurence Beck

Brian Becknell

Rolf Beetz

David Beier

Serdar Beken

Ioannis Bellos

Nadine Benador

Efrat Ben-Shalom

Albert Bensman

Carsten Bergmann

Aurelia Bertholet-Thomas

Girish Chandra Bhatt

Rajendra Bhimma

Lorenzo Biassoni

Daniel Bichet

Manuel David Bilkis

Anna Kristina Bjerre

Erica C. Bjornstad

Petter Bjornstad

Neal Blatt

Markus Bleich

Anthony Bleyer

Joshua Blinder

Detlef Bockenhauer

Arend Bokenkamp

Melvin Bonilla-Felix

Marjolein Bonthuis

Dagmara Borzych-Dużałka

Antonia Bouts

Olivia Boyer

Per Brandström

John Brandt

Michael C. Braun

Nilzete L. Bresolin

Eileen D. Brewer

Luc P. Brion

Patrick Brophy

Alexandra Bruel 
Timothy Bunchman

Nilgün Çakar

Yasar Caliskan

Chris J. Callaghan

Alexandra Cambier

Francisco Cano

Nur Canpolat

Gabriel Cara-Fuentes

Joann Carlson

J. Bryan Carmody

Simon Carter

Serkan Fazlı Celik

Vimal Chadha

Yu Hin Eugene Chan

Rahul Chanchlani

Deepa Chand

Jayanthi Chandar

Arlene Chapman

Olga Charnaya

Abanti Chaudhuri

Blanche Chavers

Cheng-Hsun Chiu

Min Hyun Cho

Elizabeth Steinberg Christofferson

Annabelle Chua

Donna J. Claes

Stephanie L. Clark

David Clive

Paula Alejandra Coccia

Pierre Cochat

Manuela Colucci

Giacomo Colussi

Rosanna Coppo

Dina Cortes

Giorgio Costagliola

Malcolm Coulthard

Richard Coward

Timothy Cummins

Amit Dagan

Stephen R. Daniels

Allison Dart

Miriam Davidovits

Jesse Davidson

Anne Elsbeth Dawson

Jeroen de Baaij

Lara de Galasso

Akash Deep

Marissa J. Defreitas

Jean-Daniel Delbet

Katherine Dell

Luca Dello Strologo

Georges Deschênes

Aniruddh V. Deshpande
Prasad Devarajan

Vikas R. Dharnidharka

Hector Diaz

Jane Dickerson

Jie Ding

Bradley P. Dixon

Aditi Dokras

Jörg Dötsch

Kevin J. Downes

Mallory L. Downie

Marie-Agnès Dragon-Durey

Dorota Drozdz

Laurence Dubourg

Jan Dudley

Minh Dien Duong

Ismail Dursun

Ali Duzova

Marina Easty

Allison Eddy

Alberto Edefonti

Vidar Orn Edvardsson

Felicia Eke

Pepe Ekulu Mfutu

Ewa Elenberg

Francesco Emma

Rachel Engen

Pieter Evenepoel

Jeffrey Fadrowski

Simone N. Fagundes

Bonita Falkner

Sahar Fathallah-Shaykh

Kristen Favel

Janusz Feber

Daniel Feig

Jorge Ferraris

Maria Ferris

Lucile Figures

Guido Filler

Richard N. Fine

Michel Fischbach

Julie Fitzgerald

Frances Flinter

Joseph T. Flynn

Agnes Fogo

Thomas Alexander Forbes

Catherine Forster

Bethany J. Foster

Anna Francis

Doris Franke

Michael A. Freeman

Michael Freundlich

Aaron L. Friedman

Yaacov Frishberg 
Dana Fuhrman

Shuichiro Fujinaga

Parvesh M. Garg

Eduardo H. Garin

Vesna Garovic

Jyothsna Gattineni

Rasheed A. Gbadegesin

Simonetta Genovesi

Roshan P. George

Gian Marco Ghiggeri

Rodney D. Gilbert

Helena Gil-Peña

Charlotte Gimpel

Mario Giordano

Debbie Gipson

Katja Gist

Beatrice Goilav

Aviva Goldberg

David S. Goldfarb

Stuart L. Goldstein

Julie Goodwin

Roberto Gordillo

Dimitrios Goumenos

Frederick Grant

Larry A. Greenbaum

Jason Henry Greenberg

Rachel Greenberg

Ryszard Grenda

Emily Groopman

Jaap W. Groothoff

Oliver Gross

Jean-Pierre Guignard

Ronnie Guillet

Isabella Guzzo

Il-Soo Ha

Sandra Habbig

Maha Haddad

Dieter Haffner

Deirdre Hahn

Susan Halbach

Nynke Halbesma

Jan Halbritter

Nancy Halnon

Coral Hanevold

Christian Hanna

Mark R. Hanudel

Jerome Harambat

Matthew Harer

Pankaj Hari

Yutaka Harita

Luke Harper

Peter Harris

Lyndsay A. Harshman
Erum A. Hartung

Elizabeth Harvey

Andrew Hattersley

Wesley Hayes

Laurence Heidet

Ian Hewitt

Guillermo Hidalgo

Eka Laksmi Hidayati

Friedhelm Hildebrandt

Sangeeta Hingorani

Daishi Hirano

Jacqueline Ho

Elisabeth M. Hodson

Julien Hogan

Jennifer Holness

Masataka Honda

Stephen R. Hooper

Bernd Hoppe

Daljit Hothi

Elion Hoxha

Peter Hoyer

Sally A. Hulton

Tracy Hunley

R. Morrison Hurley

Farida Hussain

Silvia Iacobelli

Kazumoto Iijima

Yohei Ikezumi

Elizabeth Ingulli

Sandra Iragorri

Kenji Ishikura

Shuichi Ito

Mariah Jackson

Shaun Jackson

Conxita Jacobs

Lyda Jadresic

Kitty Jager

Timo Jahnukainen

Amrish Jain

Hannu Jalanko

Peter Janssens

Mervyn Jaswon

M.P. Jayakrishnan

Marija Jelusic

Jennifer G. Jetton

Rebecca J. Johnson

Catherine Joseph

Euiseok Jung

Shoji Kagami

Aadil Kakajiwala

Mahmoud Kallash

Koichi Kamei

Elaine S. Kamil 
Konstantinos Kamperis

Yogavijayan Kandasamy

Kazunari Kaneko

Katsuyoshi Kanemoto

Hee Gyung Kang

Bernard Kaplan

Gaurav Kapur

Vasiliki Karava

Jameela Kari

Belde Kasap-Demir

Frederick Kaskel

Cristin Duongba Wendel Kaspar

Chryso Pefkaros Katsoufis

Joshua Kausman

Mandy G. Keijzer-Veen

Markus J. Kemper

Sean Kennedy

Alison Kent

Bryce A. Kerlin

Ilse Kern

David Kershaw

Tanja Kersnik Levart

Myda Khalid

Ei Khin

Kathleen Kieran

Katarzyna Kilis-Pstrusinska

Gheun-Ho Kim

Jon Jin Kim

J. Christopher Kingswood

Megan J. Kirkley

Sarah Kizilbash

Noel Knops

Vera Koch

Paulo Koch Nogueira

Amy Jo Kogon

Nika Kojc

Valentina Kon

Martin Konrad

Jeffrey B. Kopp

Mikael Koskela

Anne Marie Kouri

Ania Koziell

Leah Krischock

Jill Krissberg

Rafael T. Krmar

Juhi Kumar

Reeti Kumar

Juan C. Kupferman

Kirsten Kusumi

Eberhard Kuwertz-Bröking

David M. Kwiatkowski

Przemko Kwinta
Heather Lambert

Daniel Landau

Marc B. Lande

Craig Langman

Anne-Laure Lapeyraque

Nicholas Larkins

Benjamin Laskin

Sebastiano A.G. Lava

Asaf Lebel

Jeffrey D. Lebensburger

Kathy Lee-Son

Maren Leifheit-Nestler

Kevin Lemley

Rachel Lennon

Elena Levtchenko

Daniella Levy Erez

Qiu Li

Christoph Licht

Max C. Liebau

Marc Lilien

Fangming Lin

Jen-Jar Lin

Beata S. Lipska-Ziętkiewicz

Mieczyslaw Litwin

Chantal Loirat

Sergio Camilo Lopez-Garcia

Riccardo Lubrano

Empar Lurbe

Ian Macumber

Leire Madariaga

Britta Maecker-Kolhoff

John D. Mahan

Asmaa Mahmoud

Michal Malina

Andrew Mallett

Sai Sudha Mannemuddhu

Mukta Mantan

Stephen Marks

Matko Marlais

Olivera Marsenic

Pierluigi Marzuillo

Roos Masereeuw

Susan Massengill

Antonio Mastrangelo

Douglas G. Matsell

Mina Matsuda-Abedini

Tej Mattoo

Andrew Maxted

Heather Maxwell

Kevin McBryde

James McCaffrey

Mignon I. McCulloch 
Mara Medeiros

Otto Mehls

Djalila Mekahli

Marta Melgosa Hijosa

Nabil Ziad Melhem

Cathy Lee Mendelsohn

Susan R. Mendley

Shina Menon

Hans-Joachim Mentzel

Kevin Meyers

Julian Midgley

Gregorio Milani

Dawn S. Milliner

Om P. Mishra

Alla Mitrofanova

Yosuke Miyashita

Zubin Modi

Tahagod Mohamed

Leo Monnens

Giovanni Montini

Khemchand Netaram Moorani

Naoya Morisada

Michael L. Moritz

Asha Moudgil

Marva Moxey-Mims

Melissa Muff-Luett

Raj Munshi

Heidi J. Murphy

Mohamed Mutalib

Arwa Nada

Corina Nailescu

Koichi Nakanishi

Nehad Nasef

Kathryn Ness

Carla Nester

Alicia Neu

Tryggve Neveus

Derek Ng

Tom Nijenhuis

Damien Noone

Peter Nourse

Kandai Nozu

Jun $\mathrm{Oh}$

Takayuki Okamoto

Daryl Okamura

Eduardo Oliveira

Abiodun Omoloja

Ali Mirza Onder

Louise Oni

Michiel J.S. Oosterveld

Nelson Orta

Edgar Otto
Fatih Ozaltin

Seza Ozen

Fabio Paglialonga

Paolo Palma

Neil Paloian

Aakash Pandita

Lars Pape

Cyrielle Parmentier

Paloma Parvex

Chris Passero

Hiren Patel

Antigone Pavlaki

Amira Peco-Antić

Marco Pennesi

Mark Peters

Stefano Picca

Sergio Veloso Brant Pinheiro

Maury Pinsk

Kwanchai Pirojsakul

Caroline Platt

Lucy Plumb

Martin Pohl

Cesare Polito

Ari H. Pollack

Martin R. Pollak

Anthony A. Portale

Hans Pottel

Manuel Praga

Narayan Prasad

Evgenia Preka

Martina Prelog

Larisa Prikhodina

Laura Guimarães Primo

Cozumel S. Pruette

Uwe Querfeld

Raymond Quigley

Catherine Quinlan

Rupesh Raina

Patricio E. Ray

Brian Rayner

Jaime Manuel Restrepo

György S. Reusz

Michelle N. Rheault

Pornpimol Rianthavorn

Zaccaria Ricci

Jurgen Rissland

Syed Rizvi

Bernardo Rodríguez-Iturbe

Derek J. Roebuck

Rajeeve Rohatgi

Rodrigo Romao

Norman D. Rosenblum

David Rozansky 
Christoph Rudin

Krisztina Rusai

Bassam Saeed

Moin A. Saleem

Eloise Salmon

Matthew Sampson

Susan Samuel

Joshua Samuels

Cheryl P. Sanchez

Alison Sanders

Keia Sanderson

Ibrahim Sandokji

Simone Sanna-Cherchi

Maria Jose Santiago

Fernando Santos

David Sas

Mai Sato

Paul Saultier

Judy Savige

Happy Sawires

John Andrew Sayer

Joost Schanstra

Francesco Schena

Karl Peter Schlingmann

Claus Peter Schmitt

Ute Scholl

Michiel F. Schreuder

Andrew Schwaderer

Yogesh Scindia

Wacharee Seeherunvong

Tomas Seeman

Jeffrey L. Segar

Michael E. Seifert

Mouin Seikaly

David T. Selewski

Skaiste Sendzikaite

Sanjeev Sethi

Christine B. Sethna

Kristen Sgambat

Lokesh Shah

Nader Shaikh

Ron Shapiro

Amin S. Sharif

Ajay P. Sharma

Ankit Sharma

Vanessa Shaw

Qian Shen

Mohan Shenoy

Yuko Shima

Jae Il Shin

Stella Shin

Masahiro Shiraishi

Giridhar Mysore Shivaram
Rukshana Shroff

Ana Cristina Simões e Silva

Giacomo Simonetti

Pamela S. Singer

Aditi Sinha

Manish Sinha

Ekaterini Siomou

Sofia Sjöström

Roderick Skinner

Cara Slagle

William Smoyer

Maria Fernanda Soares

Neveen A. Soliman

Michael J.G. Somers

Megan McFerson SooHoo

Andrew Michael South

Oguz Soylemezoglu

John David Spencer

Poyyapakkam Srivaths

Stella Stabouli

Jessica Lauren Stahl

Irina Stanasel

Aleksandra Stankovic

Michelle Starr

Constantinos J. Stefanidis

Deborah Stein

Vesna Stojanović

Brian Ross Stotter

Suroj Supavekin

Megan Sutherland

Scott M. Sutherland

Sarah Swartz

Rita D. Swinford

Jordan Symons

Gregory Tasian

Jess G. Thoene

Jason Thomas

Kay Thomas

Burkhard Tönshoff

Rezan Topaloglu

Roser Torra

Vincente E. Torres

Howard Trachtman

Cheryl L. Tran

Avram Z. Traum

Agnes Trautmann

Hernan Trimarchi

Peter Trnka

Richard S. Trompeter

Jonathan P. Troost

Anne Tsampalieros

Yincent Tse

Kjell Tullus 
Katherine Twombley

Kay Tyerman

Osamu Uemura

Tim Ulinski

Kiran Upadhyay

Lambertus van den Heuvel

Michael van Husen

Steven Van Laecke

Claudia R.C. van Roeyen

Johan Vande Walle

Judith Sebestyen VanSickle

Charles Varnell

Tetyana L. Vasylyeva

Floor Veltkamp

Priya Sara Verghese

Enrico Verrina

Enrico Vidal

Sofia Visuri

Asaf Vivante

Marina Vivarelli

Oded Volovelsky

Alexander von Gontard

Christian von Schnakenburg

Dean Liam Wallace

Stephen Walsh

Mandy Wan

Chia-Shi Wang

Bradley A. Warady

Aoife Waters

Donald J. Weaver, Jr.

Lutz T. Weber

Stefanie Weber

Darcy Kate Weidemann

Thomas Welch

Scott E. Wenderfer
Patricia Liu Weng

Katherine Wesseling-Perry

Rik Westland

Aaron Wightman

Alexandra Wilhelm-Bals

Paul Winyard

Matthias Wolf

Craig Wong

Cynthia Wong

William Wong

Elke Wühl

Robert J. Wyatt

Hong Xu

$\mathrm{Xin} \mathrm{Xu}$

Monica Yamauti

Stephen Yang

Hui-Kim Yap

Hyung Eun Yim

Elisa Ylinen

Ihor V. Yosypiv

Justin Zachariah

Rima S. Zahr

Dominic Zanaboni

Marcin Zaniew

Israel Zelikovic

Qing Zhou

Peter Zipfel

Miroslav Zivicnjak

Aleksandra Zurowska

Danuta Zwolinska

Publisher's note Springer Nature remains neutral with regard to jurisdictional claims in published maps and institutional affiliations. 